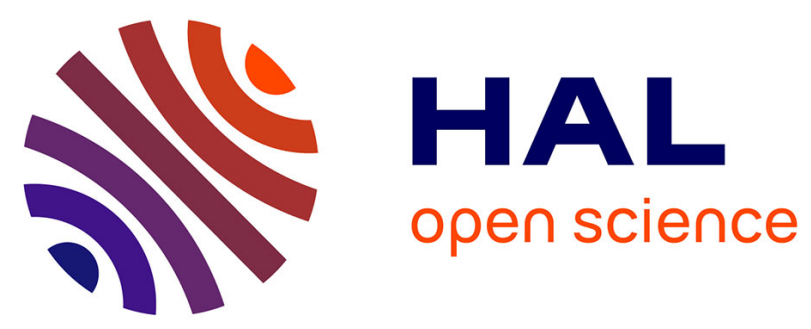

\title{
Prevalence of oral HPV infection among healthy individuals and head and neck cancer cases in the French West Indies
}

Aviane Auguste, Stanie Gaete, Cecile Herrmann-Storck, Leah Michineau, Clarisse Joachim, Jacqueline Deloumeaux, Suzy Duflo, Danièle Luce

\section{To cite this version:}

Aviane Auguste, Stanie Gaete, Cecile Herrmann-Storck, Leah Michineau, Clarisse Joachim, et al.. Prevalence of oral HPV infection among healthy individuals and head and neck cancer cases in the French West Indies. Cancer Causes and Control, 2017, 28 (11), pp.1333-1340. 10.1007/s10552-0170966-z . hal-01659247

\section{HAL Id: hal-01659247 https://hal-univ-rennes1.archives-ouvertes.fr/hal-01659247}

Submitted on 31 Jan 2020

HAL is a multi-disciplinary open access archive for the deposit and dissemination of scientific research documents, whether they are published or not. The documents may come from teaching and research institutions in France or abroad, or from public or private research centers.
L'archive ouverte pluridisciplinaire HAL, est destinée au dépôt et à la diffusion de documents scientifiques de niveau recherche, publiés ou non, émanant des établissements d'enseignement et de recherche français ou étrangers, des laboratoires publics ou privés. 
Prevalence of oral HPV infection among healthy individuals and head and neck cancer cases in the French West Indies

Authors: Aviane Auguste ${ }^{1,2}$, Stanie Gaëte ${ }^{3}$, Cécile Herrmann-Storck ${ }^{4}$, Leah Michineau ${ }^{1,2}$, Clarisse Joachim $^{5}$, Jacqueline Deloumeaux ${ }^{3,6}$, Suzy Duflo ${ }^{7}$, Danièle Luce $* 1,2$

\section{Affiliations}

${ }^{1}$ INSERM U 1085- IRSET (Research Institute for Environmental and Occupational Health), Pointe-à-Pitre, Guadeloupe, France)

${ }^{2}$ University of Rennes 1 , Rennes, France

${ }^{3}$ Centre de Ressources Biologiques de Guadeloupe, Pointe-à-Pitre, Guadeloupe, France

${ }^{4}$ Laboratory of Microbiology, University Hospital of Guadeloupe, Pointe-à-Pitre, Guadeloupe

${ }^{5}$ Martinique Cancer Registry, UF 1441 Registre des cancers, Pôle de Cancérologie Hématologie Urologie Pathologie, University Hospital of Martinique, Fort-de-France, Martinique, France

${ }^{6}$ General Cancer Registry of Guadeloupe, University Hospital of Guadeloupe, Pointe-à-Pitre, Guadeloupe, France

${ }^{7}$ Department of Oto-Rhino-Laryngology and Head and Neck Surgery, University Hospital of Guadeloupe, Pointe-à-Pitre, Guadeloupe, France

\section{Author's e-mail addresses:}

Aviane Auguste: aviane_auguste@inserm.fr

Stanie Gaëte: stanie.gaete@chu-guadeloupe.fr

Cecile Herrmann-Storck: cecile.herrmann@chu-guadeloupe.fr

Léah Michineau: leah.michineau@inserm.fr

Clarisse Joachim: clarisse.joachim@chu-martinique.fr

Jacqueline Deloumeaux: jacqueline.deloumeaux@ @hu-guadeloupe.fr

Suzy Duflo: suzy.duflo@ chu-guadeloupe.fr

Danièle Luce: daniele.luce@inserm.fr

*Correspondence and requests for reprints to:

Danièle Luce, $\mathrm{PhD}$

Inserm U 1085 - Irset

Faculté de Médecine

Campus de Fouillole

BP 145

97154 Pointe-à-Pitre

E-mail: daniele.luce@inserm.fr.

Words count for the main-text: 3413

Word count of abstract: 265 


\begin{abstract}
Purpose: Human papillomavirus (HPV) is known to play a role in the development of head and neck squamous cell carcinomas (HNSCC) and to date, no study has reported on the association between oral HPV infection and HNSCC in the Caribbean. The objective was to determine the prevalence of oral HPV infection in the French West Indies (FWI), overall and by HPV genotypes, among HNSCC cases and healthy population controls.
\end{abstract}

Method: We used data from a population-based case-control study conducted in the FWI. The prevalence of oral HPV was estimated separately among 100 HNSCC cases (mean age 59 years) and 308 population controls (mean age 57 years). Odds ratios (OR) and 95\% confidence intervals (CI) were estimated using a logistic regression adjusting for age, sex, tobacco and alcohol consumption, to assess the association between oral HPV infection and HNSCC.

Results: Prevalence of oral HPV infections was $26.0 \%$ in controls (30\% in men and $14 \%$ in women) and $36.0 \%$ in HNSCC cases (36\% in men, 33\% in women). HPV52 was the most commonly detected genotype, in cases and in controls. The prevalence of HPV16, HPV33 and HPV51 were significantly higher in cases than in controls $(p=0.0340, p=0.0472$ and 0.0144 respectively). Oral infection with high-risk HPV was associated with an increase in risk of HNSCC $(\mathrm{OR}=1.99,95 \%$ CI $0.95-4.15)$. HPV16 was only associated with oropharyngeal cancer $(\mathrm{OR}=16.01,95 \%$ CI $1.67-153.64)$.

Conclusion: This study revealed a high prevalence of oral HPV infection in this middle-aged Afro-Caribbean population, and a specific distribution of HPV genotypes. These findings may provide insight into HNSCC aetiology specific to the FWI. 


\section{Introduction}

Head and neck cancer remains a major public health problem worldwide. In the Caribbean, the estimated age-standardized (world) incidence rates for 100,000 person-years in 2012 for cancer of the lip, oral cavity, larynx and pharynx combined are 16.8 in men and 3.7 in women, similar to incidence rates in the United States (men: 16.6; women: 5.4), but higher than in Central (men: 7.8; women: 2.6) or South America (men: 13.9; women 3.8) (1). Guadeloupe and Martinique are two French overseas territories in the French West Indies (FWI). The population consists primarily of persons of African descent (about 85\%). Incidence rates of head and neck cancer in men are 25.5 per 100,000 in Guadeloupe and 15.8 per 100,000 in Martinique. Despite being lower or of the same order of magnitude as that of mainland France (22.7 per 100,000), a well-known high incidence area, these rates are among the highest in the Caribbean islands. Particularly, for pharyngeal cancer (excluding nasopharynx), Martinique (6.0 per 100,000) and Guadeloupe (6.2 per 100,000) have the top two highest incidence rates among men in the Caribbean (1). The reasons for this relatively high incidence remain unclear. Tobacco smoking and alcohol drinking are the major risk factors for these cancers. However, a recent survey has shown that tobacco and alcohol consumption are much lower in the FWI than in mainland France (2).

Human papillomavirus (HPV) is known to play a role in the development of head and neck squamous cell carcinomas (HNSCC). There are many HPV genotypes, which all have varying levels of carcinogenic capacities, ranging from no risk to high risk. HPV16 is a recognized risk factor for oropharyngeal and base of the tongue cancer, but the evidence remains inadequate for the role of other HPV types or the association between HPV and other subsites of HNSCC (3). In addition, significantly better clinical outcomes have been demonstrated in 
patients with HPV-related oropharyngeal cancer, whereas no consistent results were found for non-oropharynx subsites (4-6). Knowing the distribution of HPV in the population is therefore a great concern for the prevention and control of HNSCC in the region. The prevalence of HPV infection, the distribution of HPV genotypes and the proportion of head and neck cancers caused by HPV may vary substantially between different geographical regions (7-9). To this date, no study has been conducted to address the prevalence of oral HPV infection in the FWI; furthermore, little data are available in the Caribbean. The objective of this report was primarily to determine the prevalence of oral HPV infection in the FWI population and describe the distribution of the different genotypes among HNSCC cases and healthy individuals. In addition, we evaluated the association between HPV-integratedDNA detected in saliva and the risk of developing HNSCC.

\section{Methods}

\section{Study population, data and specimen collection}

The present report is based on data obtained from a population-based case-control study, which was conducted in the two overseas French regions in the FWI, Martinique and Guadeloupe. The study is an extension of a large nationwide case-control study, the ICARE study, which has already been conducted in ten French regions covered by a cancer registry (10). The study in the FWI used the same protocol and questionnaire, described in details elsewhere (10), with some adaptations to the local context. Eligible cases were patients residing in the FWI, suffering from a primary, malignant tumour of the oral cavity, pharynx, sinonasal cavities and larynx (International Classification of Diseases, 10th Revision, codes C00-C14; C30-C32) of any histological type, aged between 18 and 75 years old at diagnosis, newly diagnosed and histologically confirmed between April 1, 2013 and June 30, 2016. The 
inclusion of cases was performed with the collaboration of the cancer registries of Martinique and Guadeloupe. A procedure was set up to expedite case identification, in order to reduce the delay between diagnosis and interview of cases. Cases were identified through active search, by regular contacts and visits to the pathology laboratories and hospital departments that usually diagnose and treat head and neck cancers. A list of these laboratories and hospital departments was established by each registry, based on data of the previous years. The control group was selected from the general population of the FWI by random digit dialling, using incidence density sampling method. In each region (Guadeloupe or Martinique), controls were frequency matched to the cases by sex and age. Additional stratification was used to achieve a distribution by socioeconomic status among the controls comparable to that of the general population.

Cases and controls were interviewed face-to-face with a standardized questionnaire including in particular sociodemographic characteristics and lifetime tobacco and alcohol consumption. Participants were asked to provide a saliva sample, using the Oragene ${ }^{\circledR}$ OG-500 kit (DNA Genotek). Samples were sent to the Biological Resource Centre of Guadeloupe for storage at $24^{\circ} \mathrm{C}$. Oragene ${ }^{\circledR}$ saliva specimen may be stored for at least 5 years at room temperature without DNA degradation (11).

Among 257 cases identified as potentially eligible, $192(74.7 \%)$ agreed to participate and were interviewed. Among them, after diagnosis review, 22 did not meet the inclusion criteria. Among the remaining 170 cases, $114(72.3 \%)$ had provided a saliva sample. Among the 497 eligible controls, $405(81.5 \%)$ answered the questionnaire and among them $311(76.2 \%)$ provided a saliva sample. Each subject included in the study gave a written and informed consent. In order to protect the confidentiality of personal data, the questionnaire included only an identification number, without any nominative information. The same identification 
number was used for biological specimen. The link between the name and the identification number (to the exclusion of any other data) was kept by the cancer registry of the area where the subject was interviewed.

The study was approved by the Institutional Review Board of the French National Institute of Health and Medical Research and by the French Data Protection Authority.

\section{DNA extraction}

The extraction of DNA was manually performed on saliva samples. Genomic DNA extraction was carried out using prepIT ${ }^{\circledR} \cdot \mathrm{L} 2 \mathrm{P}$ reagent. The samples were mixed and incubated overnight (16 hours) at $50^{\circ} \mathrm{C}$ to ensure that DNA was released and that nucleases were permanently inactivated. Addition of the prepIT ${ }^{\circledR} \cdot \mathrm{L} 2 \mathrm{P}$ reagent revealed all impurities and the DNA in the supernatant was precipitated by adding EtOH 100\%. The DNA was washed and the pellet re-suspended in a solution of DNA Hydration (Qiagen $\left.{ }^{\circledR}\right)$ and then stored at $-20^{\circ} \mathrm{C}$.

\section{HPV detection and genotyping}

The detection of HPV was performed with the INNO-LiPA $®$ kit, which allows the detection of the following genotypes: HPV16, HPV18, HPV31, HPV33,HPV35, HPV39, HPV45, HPV51, HPV52, HPV56, HPV58, HPV59, HPV68 (High-risk), HPV26, HPV53, HPV66, HPV70, HPV73, HPV82 (Probable high-risk), HPV06, HPV11, HPV40, HPV42, HPV43, HPV44, HPV54, HPV61, HPV81 (Low-risk), HPV62, HPV67, HPV83, HPV89 (Other). The INNO-LiPA HPV genotyping assay is based on the SPF10 consensus primer system to amplify a $65 \mathrm{bp}$ fragment of the L1 region of the HPV genome (12). The assay was carried out according to the manufacturer's instructions (INNO-LiPA HPV Genotyping Extra; Innogenetics, Ghent, Belgium). 
The amplification was performed using SPF10 primers, with adding primers to amplify the human HLA-DPB1 region for having a control of the DNA quality at the same time. The amplification was performed in a reagent mixture containing biotinylated primers in buffer with dNTP/dUTP mix, $\mathrm{MgCl} 2, \mathrm{NaN} 3$ as preservative, AmpliTaq Gold® polymerase and uracil-N-glycosylase. Before amplification, DNA was added. All PCR reactions were performed with a positive and a negative control. The biotinylated PCR products were genotyped by denaturation and hybridization on nitrocellulose strips followed by a stringent wash. After the addition of the conjugate and the substrate, a colorimetric analysis revealed all the genotypes present in the sample. The hybridization process was automatically performed on the Autoblot $3000 \mathrm{H}$; at the end, the strip was fixed on a support to read the HPV genotypes lines correspondence.

Due to the presence of primers that amplify all genotypes simultaneously, if there was more competition between particular genotypes, only the presence of a broad range of HPV was detected with the line control HPV1 and/or the line control HPV2. This kind of sample was notified HPV-positive without specifying the genotype. These samples were classified as "undetermined" and were included in the calculation for the prevalence of oral HPV infection regardless of the genotype. However, these samples were excluded from the individual genotype analysis.

\section{Statistical analysis}

The analysis was restricted to squamous cell carcinomas (100 cases). For three controls, the quality of the specimen collected was considered inadequate for HPV detection. Our analysis finally included 408 subjects among which 100 were cases and 308 were controls. A univariate analysis was performed to describe the characteristics of the subjects included in 
the study. A Chi-squared test was used to test the association between these characteristics and HNSCC. The prevalence of oral HPV infections was estimated separately among the HNSCC cases and the controls. Subjects with DNA of at least one HPV type detected in saliva sample are referred to as HPV-positive. This was then repeated for the different category of carcinogenic risk (high-risk, probable high-risk, low-risk and other) and the various HPV genotypes. The prevalence was also calculated for different categories of the subject characteristics: age, sex recruitment site, tobacco smoking (ever vs never), alcohol drinking (ever daily drinker, i.e. at least one glass per day during at least one year; never daily drinker). The prevalence calculation was performed by determining the absolute number of HPV-positive cases/controls and then dividing by the total number of cases/controls included in the study and $95 \%$ CI were calculated. The association between the oral HPV infection and the occurrence of HNSCC was assessed by estimating odds ratios (OR) and 95\% confidence intervals (CI) using a logistic regression model adjusting for age, sex, tobacco smoking and alcohol drinking. An exact Fisher test was performed to assess this association for each HPV genotype individually. Tests giving a p-value lower than $5 \%$ were considered statistically significant. Statistical analysis was performed using SAS 9.4 software (SAS Institute, Carry, NC USA).

\section{Results}

\section{Characteristics of study population}

Table 1 provides a description of selected characteristics of the cases and controls included in the study. The mean age was similar in both cases and controls (59 years and 57 years respectively), but the age distribution differed $(\mathrm{p}=0.0163)$. The proportion of women was significantly greater in the control group $(\mathrm{p}=0.0026)$. The proportion of subjects by region did 
not differ between cases and controls $(\mathrm{p}=0.9311)$. As expected, tobacco smoking $(\mathrm{p}<0.0001)$ and alcohol drinking $(\mathrm{p}<0.0001)$ were more frequent among cases than among controls.

\section{Oral HPV prevalence}

Table 2 provides the oral HPV prevalence by age group, sex, recruitment site, tobacco smoking and alcohol drinking for HNSCC cases and controls separately. Overall, oral HPV was found in $36.0 \%$ (CI95\%=[27.6 - 47.2\%] of the cases and 26.0\% (CI95\%=[21.2-31.3\%] of the controls. The subjects aged between 55 and 64 years had the highest prevalence of HPV in both cases and controls $(48.8 \%$; CI95\% $=[33.35-65.5 \%]$ and 35.8\%; CI95\%= $[26.2$ - $46.3 \%$ ] respectively), when compared to the other age groups. Among the controls, oral HPV was found to be twice as prevalent in men as in women, whereas the prevalence was similar in men and women among the cases. A significantly greater HPV prevalence was observed in Guadeloupe than in Martinique regardless of the cancer status.

Table 3 shows the prevalence of high-risk, probable high-risk, low-risk and other HPV types, and of the individual HPV genotypes. The prevalence of high-risk HPV types was found to be $23.3 \%$ in the cases and $10.7 \%$ in the controls $(\mathrm{p}=0.005)$. Concerning the other risk categories (probable high-risk, low-risk and other), the prevalence did not differ significantly between cases and controls. The most frequent HPV genotypes detected among the controls were HPV66 (5.0\%) and HPV52 (4.3\%). Whereas the genotypes HPV52, HPV56 and HPV16 were the most frequent among the cases $(8.9 \%, 5.6 \%$ and $4.4 \%$ respectively). The prevalence of HPV16, HPV33 and HPV51 were significantly higher in cases than in controls $(\mathrm{p}=0.0340$, $\mathrm{p}=0.0472$ and 0.0144 respectively).

We also looked at the HPV prevalence and genotype distribution by cancer site. The following sites were distinguished: oral cavity (oral tongue, gum, mouth, floor of mouth, lips; 
22 cases), oropharynx (base of tongue, tonsil, other parts of the oropharynx; 41 cases), larynx/hypopharynx (23 cases) and other sites (sinonasal cavities 4 cases). The prevalence of oral HPV infection was $34.1 \%, 32.0 \%$ and $34.6 \%$ in cancers of the oropharynx, oral cavity and larynx/hypopharynx respectively. The prevalence of high-risk HPV was similar in oropharyngeal $(22.0 \%)$ and non-oropharyngeal $(23.0 \%)$ cancer cases. HPV-16 was detected exclusively in oropharyngeal cancer cases (4 cases). The three cases positive for HPV33 were 2 oropharyngeal cancers cases and one oral cavity cancer. HPV51 was detected in one oropharyngeal cancer, in one oral cavity cancer and in one laryngeal cancer. Other HPV types were not found to be associated with specific cancer sites.

\section{Association between oral HPV and HNSCC}

Table 4 give the results of the logistic regression adjusted for age, sex, tobacco smoking and alcohol drinking, modelling the risk of developing a HNSCC. The overall HPV infection regardless of the level of carcinogenicity was not found significantly associated to HNSCC. Oral infection with high-risk HPV was associated with a two-fold increase in risk of HNSCC $(\mathrm{OR}=1.99,95 \%$ CI $[0.95-4.15])$. The association between HPV16 and HNSCC risk $(\mathrm{OR}=6.2495 \% \mathrm{CI}[0.76-51.35])$ was limited to oropharyngeal cancer $(\mathrm{OR}=16.0195 \% \mathrm{CI}$ $[1.67-153.64])$.

\section{Discussion}

This is the first study in the Caribbean reporting on oral HPV infection in both HNSCC cases and healthy individuals of African descent. We found an overall HPV prevalence of $36 \%$ among HNSCC cases, with little variation by cancer site. Our results are globally compatible with those of a recent meta-analysis that estimated for tumours from patients of Central and South America an overall HPV DNA prevalence of 33.1\% (95\% CI 15.4-53.6) for cancers of 
the oral cavity, $14.9 \%$ (95\% CI 5.6-27.0) for oropharyngeal cancer and 32.2\% (95\% CI 15.551.4) for laryngeal/hypopharyngeal cancer (7). Another meta-analysis of HPV prevalence in tumours from HNSCC patients of African descent reported a prevalence of $17 \%$ (CI95\%= [8.8-27.0\%]), higher among oropharyngeal cancers (31.5\%) than in non-oropharyngeal cancers $(14.5 \%)(13)$. The prevalence of oral HPV infection in our study was similar for oropharyngeal cancer (29.3\%), but was higher for other cancer sites $(28.6 \%)$. In recent casecontrol studies on HNSCC (14-18), the prevalence of HPV infection in the oral cavity varied from $19 \%$ to $49 \%$ for all HNSCC, and from $37 \%$ to $61 \%$ for oropharyngeal cancers. Contrary to most other studies, HPV16 was not the most frequently detected HPV type in our study, resulting in a low prevalence of HPV16 among cases. However, the prevalence of HPV16 was similar for oropharyngeal cancer (10\%) to that observed in Central and South America (14.5\%) (7).

We took advantage of the controls recruited in this study to estimate the prevalence of oral HPV infections in the general population of the FWI. The overall prevalence for the two regions was $26 \%$. The prevalence that we estimated in our study was on average greater than in previous studies reporting on oral HPV prevalence in healthy individuals from different geographic regions. In a literature review, oral HPV infection prevalence was estimated to be 4.5\% (95\% CI: 3.9-5.1\%) overall, 3.5\% (95\% CI: 3.0-4.1\%) for high-risk HPV types and 1.3\% (95\% CI: 1.0-1.7\%) for HPV16(9). Our control group was however frequency matched to the cases by age and sex, which skewed the results towards older ages and male gender. Furthermore, the small number of subjects below 45 years made it difficult to estimate precisely the HPV prevalence for this category. Consequently, the overall prevalence in our sample is likely to overestimate the prevalence in the general population of the FWI, but provides a fairly reliable estimate of the prevalence in the population of the FWI over 45 
years of age. The prevalence of oral HPV infection in our controls is higher than that recently estimated in the US, in men $(10.1 \%)$ and in women (3.6\%), even in the older age categories (55-59: $11.2 \%, 60-6411.4 \%$ ). The peak prevalence among individuals aged 55 to 64 years and the higher prevalence in men observed in this study are consistent with our results (19). Our estimate is also higher than in a multinational sample of healthy men $(6.1 \%$ in men aged 55-74 years)(20). This distinct difference in prevalence was observed even in a study conducted in another Caribbean population. The prevalence in women in our control group (14.6\%) was more than that of another study reporting on the oral HPV in Tobagonian women $(6.6 \%)$ who were however younger (median age 42 years) than the women in our study (21). In controls of case-control studies on HNSCC (14-18), who had an age and sex distribution similar to our controls, the prevalence of oral HPV infection varied from 5\% to $17.3 \%$. As noted above for the cases, in our controls also HPV16 was not the predominant genotype, and the high HPV prevalence observed in our control group was mainly due to genotypes other than HPV16. It is worth noting that a high prevalence of cervical infection with HPV genotypes other than 16 or 18 was also found among healthy women in Guadeloupe (22).

In our study, overall oral HPV was not found to be significantly associated with HNSCC. This absence of association was consistent with another study which found that the proportion of HPV-positive was almost identical between cases and controls (23). Other studies reported significant associations between overall oral HPV infections and HNSCC, in particular for oral cancer and oropharyngeal cancer $(14,15,18)$. The lack of association with overall HPV infection in our study may be due to the specific distribution of HPV genotypes in our population. Indeed, we found a borderline significant association between high-risk HPV and HNSCC, and a strong and significant association between HPV16 and oropharyngeal cancer. The latter result is consistent with previous studies $(3,18,24)$. Our study revealed also a larger 
proportion of HPV33 and HPV51 among the HNSCC cases than the controls. The associations with HPV33,HPV51 and HNSCC were not observed in previous studies $(15,20,21)$. In addition, HPV51 was found exclusively in cases and this could provide a good lead for subsequent studies. These findings could be useful to assess the potential efficiency of current HPV vaccination strategies for the prevention of HNSCC in these regions.

We are aware that our study has some limitations that need to be accounted for when interpreting the data. Firstly, the HPV was detected using saliva samples. This means that the HPV infections were prevalent and we had no means of determining whether or not the HPV infection preceded the HNSCC diagnosis. In addition, we had no information of HPV tumour status. However, several studies have reported a good correlation between HPV DNA detection in tumour tissue and saliva rinse $(17,25,26)$, and the use of saliva samples was also shown to be sensitive and specific for p16-positive oropharyngeal tumours (27). Secondly, the relatively small number of HNSCC cases hampered detailed analyses by cancer site and HPV genotype. Selection bias may not be excluded but is thought to be minimal in the present study. The distribution by sex, age and cancer sites of the cases included in our study was similar to that of the cases in the local cancer registries. Our study population can thus be considered representative of the HNSCC cases. The method used to select the control group was previously demonstrated to yield unbiased samples and the controls could be considered representative of the general population of similar age and sex (10). Furthermore, this is one of the very few case-control studies which has investigated the role of oral HPV infection in men and women of African descent and will allow comparison with French HPV data to investigate potential racial disparities between these populations (28). This study will add valuable data supporting the prevention and control of HNSCC in the people of this ethnic group. 


\section{Conclusion}

To conclude, the prevalence of oral HPV infection in the French West Indies is $26.0 \%$ among healthy individuals and $36.0 \%$ in HNSCC patients. The detection of overall oral HPV was not found to influence significantly the occurrence of HNSCC. However, high-risk HPV and the individual genotypes HPV16, HPV33 and HPV51 increased the risk of HNSCC. These findings are particularly interesting because they give valuable leads on the aetiology of these cancers in the FWI. Subsequent analyses will examine the potential interactions with traditional risk factors.

\section{Conflict of interest statement}

The authors have no conflict of interest

\section{Acknowledgements}

We would like to thank our clinical research associates Lucina Lipau and Audrey Pomier for their help in data collection. Aviane Auguste was supported by a grant from the "Ligue contre le Cancer, comité d'Ille-et-Vilaine" for this work. This study was funded by the French National Cancer Institute (Institut National du Cancer) and the Cancéropôle Ile-de-France.

\section{Ethical approval}

French Data Protection Authority (CNIL, Commission Nationale de l'Informatique et des Libertés) n DR-2015-2027; IRB INSERM n01-036 


\section{References}

1. Ferlay J, Soerjomataram I, Ervik M, Dikshit R, Eser S, Mathers C, et al. GLOBOCAN 2012 v1.0, Cancer Incidence and Mortality Worldwide: IARC CancerBase No. 11 [Internet]. International Agency for Research on Cancer. 2013 [cited 2017 May 30]. Available from: http://globocan.iarc.fr

2. Jean-Baptiste Richard. Premiers résultats du Baromètre santé DOM 2014 - Résultats détaillés selon le DOM, l'âge et le sexe - 2015. 2015.

3. Gillison ML, Alemany L, Snijders PJF, Chaturvedi A, Steinberg BM, Schwartz S, et al. Human Papillomavirus and Diseases of the Upper Airway: Head and Neck Cancer and Respiratory Papillomatosis. Vaccine. 2012 Nov 20;30:F34-54.

4. Fakhry C, Westra WH, Li S, Cmelak A, Ridge JA, Pinto H, et al. Improved survival of patients with human papillomavirus-positive head and neck squamous cell carcinoma in a prospective clinical trial. J Natl Cancer Inst. 2008 Feb 20;100(4):261-9.

5. Ang KK, Harris J, Wheeler R, Weber R, Rosenthal DI, Nguyen-Tân PF, et al. Human papillomavirus and survival of patients with oropharyngeal cancer. N Engl J Med. 2010 Jul 1;363(1):24-35.

6. Ragin CCR, Taioli E. Survival of squamous cell carcinoma of the head and neck in relation to human papillomavirus infection: review and meta-analysis. Int J Cancer. 2007 Oct 15;121(8):1813-20.

7. Ndiaye C, Mena M, Alemany L, Arbyn M, Castellsagué X, Laporte L, et al. HPV DNA, E6/E7 mRNA, and p16INK4a detection in head and neck cancers: a systematic review and meta-analysis. Lancet Oncol. 2014 Nov;15(12):1319-31.

8. Kreimer AR, Clifford GM, Boyle P, Franceschi S. Human Papillomavirus Types in Head and Neck Squamous Cell Carcinomas Worldwide: A Systematic Review. Cancer Epidemiol Biomarkers Prev. 2005 Feb 1;14(2):467-75.

9. Kreimer AR, Bhatia RK, Messeguer AL, Gonzalez P, Herrero R, Giuliano AR. Oral Human Papillomavirus in Healthy Individuals: A Systematic Review of the Literature. Sexually Transmitted Diseases June 2010. 2010;37(6):386-91.

10. Luce D, Stücker I, ICARE Study Group. Investigation of occupational and environmental causes of respiratory cancers (ICARE): a multicenter, population-based case-control study in France. BMC Public Health. 2011 Dec 14;11:928.

11. Iwasiow RM, Desbois A, Birnboim HC. Long-term stability of DNA from saliva samples stored in the Oragene self-collection kit. DNA Genotek [Internet]. 2011; Available from: https://kyodo-inc.co.jp/english/bio/oragene/pdf/faq_dna02_001.pdf

12. Kleter B, van Doorn LJ, Schrauwen L, Molijn A, Sastrowijoto S, ter Schegget J, et al. Development and clinical evaluation of a highly sensitive PCR-reverse hybridization 
line probe assay for detection and identification of anogenital human papillomavirus. $\mathbf{J}$ Clin Microbiol. 1999 Aug;37(8):2508-17.

13. Ragin C, Liu JC, Jones G, Shoyele O, Sowunmi B, Kennett R, et al. Prevalence of HPV Infection in Racial-Ethnic Subgroups of Head and Neck Cancer Patients. Carcinogenesis. 2016 Dec 26;

14. D’Souza G, Kreimer AR, Viscidi R, Pawlita M, Fakhry C, Koch WM, et al. Case-control study of human papillomavirus and oropharyngeal cancer. N Engl J Med. 2007 May 10;356(19):1944-56.

15. Pintos J, Black MJ, Sadeghi N, Ghadirian P, Zeitouni AG, Viscidi RP, et al. Human papillomavirus infection and oral cancer: A case-control study in Montreal, Canada. Oral Oncology. 2008 Mar;44(3):242-50.

16. Anaya-Saavedra G, Ramírez-Amador V, Irigoyen-Camacho ME, García-Cuellar CM, Guido-Jiménez M, Méndez-Martínez R, et al. High association of human papillomavirus infection with oral cancer: a case-control study. Arch Med Res. 2008 Feb;39(2):189-97.

17. Tachezy R, Klozar J, Rubenstein L, Smith E, Saláková M, Šmahelová J, et al. Demographic and risk factors in patients with head and neck tumors. J Med Virol. 2009 May 1;81(5):878-87.

18. Laprise C, Madathil SA, Schlecht NF, Castonguay G, Soulières D, Nguyen-Tan PF, et al. Human papillomavirus genotypes and risk of head and neck cancers: Results from the HeNCe Life case-control study. Oral Oncol. 2017 Jun;69:56-61.

19. Gillison ML, Broutian T, Pickard RKL, Tong Z, Xiao W, Kahle L, et al. Prevalence of oral HPV infection in the United States, 2009-2010. JAMA. 2012 Feb 15;307(7):693703.

20. Kreimer AR, Villa A, Nyitray AG, Abrahamsen M, Papenfuss M, Smith D, et al. The epidemiology of oral HPV infection among a multinational sample of healthy men. Cancer Epidemiol Biomarkers Prev. 2011 Jan;20(1):172-82.

21. Ragin CCR, Wheeler VW, Wilson JB, Bunker CH, Gollin SM, Patrick AL, et al. Distinct distribution of HPV types among cancer-free Afro-Caribbean women from Tobago. Biomarkers. 2007 Jan 1;12(5):510-22.

22. Cordel N, Ragin C, Trival M, Tressières B, Janky E. High-risk human papillomavirus cervical infections among healthy women in Guadeloupe. International Journal of Infectious Diseases. 2015 Dec;41:13-6.

23. Schwartz SM, Daling JR, Doody DR, Wipf GC, Carter JJ, Madeleine MM, et al. Oral cancer risk in relation to sexual history and evidence of human papillomavirus infection. J Natl Cancer Inst. 1998 Nov 4;90(21):1626-36. 
24. D’Souza G, Kreimer AR, Viscidi R, Pawlita M, Fakhry C, Koch WM, et al. Casecontrol study of human papillomavirus and oropharyngeal cancer. New England Journal of Medicine. 2007;356(19):1944-1956.

25. Smith EM, Ritchie JM, Summersgill KF, Klussmann JP, Lee JH, Wang D, et al. Age, sexual behavior and human papillomavirus infection in oral cavity and oropharyngeal cancers. International Journal of Cancer. 2004 Feb 20;108(5):766-72.

26. Zhao M, Rosenbaum E, Carvalho AL, Koch W, Jiang W, Sidransky D, et al. Feasibility of quantitative PCR-based saliva rinse screening of HPV for head and neck cancer. Int J Cancer. 2005 Nov 20;117(4):605-10.

27. Wasserman JK, Rourke R, Purgina B, Caulley L, Dimitroulakis J, Corsten M, et al. HPV DNA in saliva from patients with SCC of the head and neck is specific for p16-positive oropharyngeal tumours. J Otolaryngol Head Neck Surg. 2017 Jan 6;46(1):3.

28. Peterson CE, Khosla S, Chen LF, Joslin CE, Davis FG, Fitzgibbon ML, et al. Racial differences in head and neck squamous cell carcinomas among non-Hispanic black and white males identified through the National Cancer Database (1998-2012). J Cancer Res Clin Oncol. 2016 Aug;142(8):1715-26. 
Table 1: Main characteristics of HNSCC cases and controls. French West Indies, 2013-2016

\begin{tabular}{|c|c|c|c|c|c|c|}
\hline \multirow{2}{*}{ Variable } & \multirow{2}{*}{ Category } & \multicolumn{2}{|c|}{ Cases $(n=100)$} & \multicolumn{2}{|c|}{ Controls $(n=308)$} & \multirow{2}{*}{ p-value } \\
\hline & & $\mathrm{n}$ & $(\%)$ & $\mathrm{n}$ & $(\%)$ & \\
\hline \multirow{3}{*}{ Age } & $<45$ & 4 & $(4.0)$ & 44 & $(14.3)$ & \\
\hline & $55-64$ & 43 & $(43.0)$ & 95 & $(30.8)$ & \\
\hline & $>=65$ & 26 & $(26.0)$ & 88 & $(28.6)$ & \\
\hline Sex & & & & & & 0.0026 \\
\hline \multirow[t]{3}{*}{ Recruitment site } & & & & & & 0.9311 \\
\hline & Martinique & 44 & $(44.0)$ & 134 & $(43.5)$ & \\
\hline & Guadeloupe & 56 & $(56.0)$ & 174 & $(56.5)$ & \\
\hline \multirow[t]{3}{*}{ Tobacco smoking } & & & & & & \\
\hline & Ever & 80 & $(80.0)$ & 106 & $(34.4)$ & $<.0001$ \\
\hline & Never & 20 & $(20.0)$ & 202 & $(65.6)$ & \\
\hline
\end{tabular}


Table 2: Prevalence of oral HPV infection by age, sex, recruitment site, tobacco and alcohol consumption among HNSCC cases and controls. French West Indies, 2013-2016

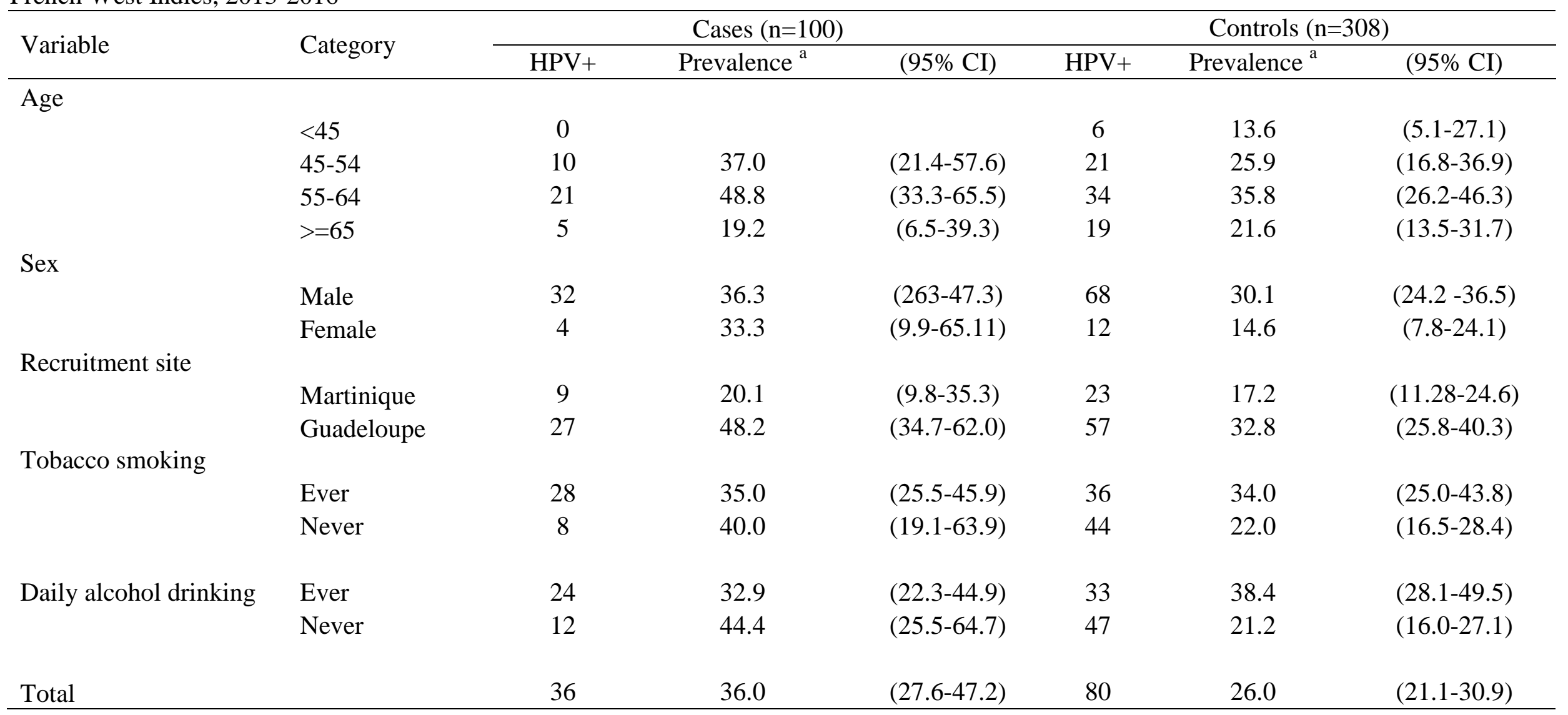

a: Prevalence calculated by dividing the number of HPV+ by the total number of subjects for a given category. 
Table 3: Prevalence of oral HPV infection by genotype among HNSCC cases and controls. French West Indies, 2013-2016

\begin{tabular}{|c|c|c|c|c|c|}
\hline \multirow{2}{*}{ Genotype $^{\mathrm{a}}$} & \multicolumn{2}{|c|}{ Cases $(n=90)$} & \multicolumn{2}{|c|}{ Controls $(n=281)$} & \multirow{2}{*}{ P-value } \\
\hline & $\mathrm{n}$ & $(\%)^{\mathrm{b}}$ & $\mathrm{n}$ & $(\%)^{\mathrm{b}}$ & \\
\hline High-risk & 21 & $(23.3)$ & 30 & $(10.7)$ & 0.0050 \\
\hline HPV16 & 4 & $(4.4)$ & 2 & $(0.7)$ & 0.0340 \\
\hline HPV18 & 2 & $(2.2)$ & 1 & $(0.4)$ & 0.1501 \\
\hline HPV31 & 2 & $(2.2)$ & 6 & (2.1) & 1 \\
\hline HPV33 & 3 & (3.3) & 1 & $(0.4)$ & 0.0472 \\
\hline HPV39 & 0 & $(0.0)$ & 1 & $(0.4)$ & 1 \\
\hline HPV45 & 1 & (1.1) & 2 & $(0.7)$ & 0.5708 \\
\hline HPV51 & 3 & (3.3) & 0 & $(0.0)$ & 0.0144 \\
\hline HPV52 & 8 & (8.9) & 12 & $(4.3)$ & 0.1116 \\
\hline HPV56 & 5 & (5.6) & 6 & $(2.1)$ & 0.1473 \\
\hline HPV58 & 0 & $(0.0)$ & 4 & $(1.4)$ & 0.5761 \\
\hline HPV59 & 1 & (1.1) & 1 & $(0.4)$ & 0.4306 \\
\hline HPV68 & 2 & $(2.2)$ & 8 & $(2.8)$ & 1 \\
\hline Probable high-risk & 6 & (6.7) & 24 & $(8.5)$ & 0.6628 \\
\hline HPV26 & 1 & (1.1) & 0 & $(0.0)$ & 0.2451 \\
\hline HPV53 & 0 & $(0.0)$ & 4 & (1.4) & 0.5761 \\
\hline HPV66 & 4 & (4.4) & 14 & $(5.0)$ & 1 \\
\hline HPV70 & 1 & (1.1) & 3 & (1.1) & 1 \\
\hline HPV73 & 0 & $(0.0)$ & 2 & $(0.7)$ & 1 \\
\hline HPV82 & 1 & (1.1) & 2 & $(0.7)$ & 0.5708 \\
\hline Low-risk & 6 & (6.7) & 13 & $(4.6)$ & 0.4258 \\
\hline HPV06 & 3 & $(3.3)$ & 3 & (1.1) & 0.1603 \\
\hline HPV42 & 1 & $(1.1))$ & 0 & $(0.0)$ & 0.2451 \\
\hline HPV44 & 1 & $(1.1)$ & 5 & $(1.8)$ & 1 \\
\hline HPV 54 & 0 & $(0.0)$ & 1 & $(0.4)$ & 1 \\
\hline HPV61 & 2 & $(2.2)$ & 2 & $(0.7)$ & 0.2529 \\
\hline HPV81 & 0 & $(0.0)$ & 3 & $(1.1)$ & 1 \\
\hline Other & 5 & (5.6) & 7 & $(2.5)$ & 0.1769 \\
\hline HPV62 & 2 & $(2.2)$ & 2 & $(0.7)$ & 0.2529 \\
\hline HPV67 & 2 & (2.2) & 3 & (1.1) & 0.6000 \\
\hline HPV83 & 0 & $(0.0)$ & 2 & $(0.7)$ & 1 \\
\hline
\end{tabular}

a: The following HPV genotypes were not detected in our sample and were omitted from the table: HPV35, HPV26, HPV11 HPV40 and HPV89.

b: Percentage calculated by dividing by the number of cases/controls. Note that because of multiple infections the individual genotype percentages do not add up to give the total amount of the risk group that they belong to.

c: P-value from exact Fisher test. 
Table 4: Association between oral HPV infection and HNSCC risk. French West Indies, 2013-2016

\begin{tabular}{|c|c|c|c|c|}
\hline HPV category & $\begin{array}{c}\text { Cases } \\
\mathrm{n} \\
\end{array}$ & $\begin{array}{c}\text { Controls } \\
\mathrm{n}\end{array}$ & $\mathrm{OR}^{\mathrm{a}}$ & $(95 \% \mathrm{CI})$ \\
\hline HPV-negative & 69 & 228 & 1 & (ref) \\
\hline Any HPV & 37 & 80 & 1.13 & $(0.63-1.99)$ \\
\hline High-risk & 21 & 30 & 1.99 & $(0.95-4.15)$ \\
\hline HPV16 & 4 & 2 & 6.24 & $(0.76-51.35)$ \\
\hline Probable high-risk & 6 & 24 & 0.42 & $(0.15-1.21)$ \\
\hline Low-risk & 6 & 13 & 1.85 & $(0.56-6.11)$ \\
\hline Other & 5 & 7 & 1.35 & $(0.33-5.63)$ \\
\hline
\end{tabular}

a: Logistic regression modelling the occurrence of HNSCC, odds ratios adjusted for age, sex, tobacco smoking and daily alcohol drinking. 